\title{
A note on a series containing the Laguerre polynomials
}

\author{
Y. S. Kim, A. K. Rathie, and R. B. Paris
}




\title{
A NOTE ON A SERIES CONTAINING THE LAGUERRE POLYNOMIALS
}

\author{
Y. S. KIM, A. K. RATHIE, AND R. B. PARIS
}

Received 05 November, 2013

Abstract. Expressions for the summation of the series involving the Laguerre polynomials

$$
S( \pm v, \pm j) \equiv e^{-x} \sum_{n=0}^{\infty} \frac{x^{n} L_{n}^{(v)}(x)}{(1 \pm v \pm j)_{n}}
$$

for any non-negative integer $j$ are obtained in terms of generalized hypergeometric functions. These results provide alternative, and in some cases simpler expressions to those recently obtained in the literature.

2010 Mathematics Subject Classification: 33C15; 33C20

Keywords: Laguerre polynomials, generalized hypergeometric functions, generalized Kummer summation theorem

\section{INTRODUCTION}

The generalized Laguerre polynomials $L_{n}^{(v)}(x)$ are encountered in many branches of pure and applied mathematics. They form an orthogonal set on $[0, \infty)$ with the weight function $x^{v} e^{-x}$, with the first three polynomials given by

$$
\begin{aligned}
& L_{0}^{(v)}(x)=1, \\
& L_{1}^{(v)}(x)=1-x+v, \\
& L_{2}^{(v)}(x)=\frac{1}{2} x^{2}-(v+2) x+\frac{1}{2}(v+1)(v+2) .
\end{aligned}
$$

In general, $L_{n}^{(v)}(x)$ can be represented as a terminating confluent hypergeometric function ${ }_{1} F_{1}$ in the form

$$
L_{n}^{(v)}(x)=\frac{(v+1)_{n}}{n !}{ }_{1} F_{1}(-n ; v+1 ; x) .
$$

Here $(a)_{n}$ denotes the Pochhammer symbol, or rising factorial, defined by $(a)_{n}=\frac{\Gamma(a+n)}{\Gamma(a)}$. 
In [2], Kim et al. obtained summation formulas for the two series involving the generalized Laguerre polynomial $L_{n}^{(v)}(x)$ given by

$$
\sum_{n=0}^{\infty} \frac{x^{n} L_{n}^{(v)}(x)}{(1 \pm v+j)_{n}}
$$

for integer $j$, where $-5 \leq j \leq 5$. Recently, Brychkov [1] has extended these results for any integer $j$. The aim of this note is to derive alternative expressions for the summation of the series

$$
S( \pm v, \pm j) \equiv e^{-x} \sum_{n=0}^{\infty} \frac{x^{n} L_{n}^{(v)}(x)}{(1 \pm v \pm j)_{n}}
$$

for any non-negative integer $j$. Our results are different from, and in some cases simpler, than those obtained in [1].

\section{THE SERIES $S(v, \pm j)$}

We start with the transformation [2, (3.5)]

$$
\begin{aligned}
e^{-x} \sum_{n=0}^{\infty} \frac{\left(a_{1}\right)_{n} \ldots\left(a_{p}\right)_{n}}{\left(b_{1}\right)_{n} \ldots\left(b_{q}\right)_{n}} & (-x y)^{n} L_{n}^{(v)}(x) \\
& =\sum_{n=0}^{\infty} \frac{(-x)^{n}}{n !} p+2 F_{q}\left[\begin{array}{c}
\left.-n,-n-v, a_{1}, \ldots, a_{p} ; y\right] \\
b_{1}, \ldots, b_{q}
\end{array}\right]
\end{aligned}
$$

where $p$ and $q$ are non-negative integers and ${ }_{p} F_{q}$ denotes the generalized hypergeometric function. In this, if we take $p=0, q=1, b_{1}=1+v+j$ and $y=-1$, then

$$
e^{-x} \sum_{n=0}^{\infty} \frac{x^{n} L_{n}^{(v)}(x)}{(1+v+j)_{n}}=\sum_{n=0}^{\infty} \frac{(-x)^{n}}{n !}{ }_{2} F_{1}\left[\begin{array}{c}
-n,-n-v \\
1+v+j
\end{array} ;-1\right] .
$$

The ${ }_{2} F_{1}$ series on the right-hand side of (2.2) can be evaluated with the help of the generalized Kummer summation theorem [3]

$$
\begin{aligned}
{ }_{2} F_{1}\left[\begin{array}{c}
a, b \\
1+a-b+j
\end{array} ;-1\right]= & \frac{2^{-a} \Gamma\left(\frac{1}{2}\right) \Gamma(b-j) \Gamma(1+a-b+j)}{\Gamma(b) \Gamma\left(\frac{1}{2} a-b+\frac{1}{2} j+\frac{1}{2}\right) \Gamma\left(\frac{1}{2} a-b+\frac{1}{2} j+1\right)} \\
& \times \sum_{r=0}^{j}(-1)^{r}\left(\begin{array}{c}
j \\
r
\end{array}\right) \frac{\Gamma\left(\frac{1}{2} a-b+\frac{1}{2} j+\frac{1}{2} r+\frac{1}{2}\right)}{\Gamma\left(\frac{1}{2} a-\frac{1}{2} j+\frac{1}{2} r+\frac{1}{2}\right)}
\end{aligned}
$$

for $j=0,1,2, \ldots$.

After some straightforward simplification, we obtain

$$
S(v, j) \equiv e^{-x} \sum_{n=0}^{\infty} \frac{x^{n} L_{n}^{(v)}(x)}{(1+v+j)_{n}}
$$




$$
\begin{aligned}
= & \frac{(-1)^{j} 2^{2 v+j} \Gamma(1+v)}{\Gamma(1+2 v+j)} \sum_{r=0}^{j}(-1)^{r}\left(\begin{array}{c}
j \\
r
\end{array}\right)\left\{\frac{\Gamma\left(v+\frac{1}{2} j+\frac{1}{2} r+\frac{1}{2}\right)}{\Gamma\left(\frac{1}{2}-\frac{1}{2} j+\frac{1}{2} r\right)}\right. \\
& \times{ }_{4} F_{5}\left[\begin{array}{c}
\frac{1}{2}+\frac{1}{2} v, 1+\frac{1}{2} v, \frac{1}{2}+v+\frac{1}{2} j+\frac{1}{2} r, \frac{1}{2}+\frac{1}{2} j-\frac{1}{2} r \\
\frac{1}{2}, \frac{1}{2}+\frac{1}{2} v+\frac{1}{2} j, 1+\frac{1}{2} v+\frac{1}{2} j, \frac{1}{2}+v+\frac{1}{2} j, 1+v+\frac{1}{2} j
\end{array} ;-x^{2}\right] \\
& -\frac{\Gamma x(1+v)}{(1+v+j)(1+2 v+j)} \frac{\Gamma\left(v+\frac{1}{2} j+\frac{1}{2} r+1\right)}{\Gamma\left(\frac{1}{2} r-\frac{1}{2} j\right)} \\
& \left.\times{ }_{4} F_{5}\left[\begin{array}{c}
1+\frac{1}{2} v, \frac{3}{2}+\frac{1}{2} v, 1+v+\frac{1}{2} j+\frac{1}{2} r, 1+\frac{1}{2} j-\frac{1}{2} r \\
\frac{3}{2}, 1+\frac{1}{2} v+\frac{1}{2} j, \frac{3}{2}+\frac{1}{2} v+\frac{1}{2} j, 1+v+\frac{1}{2} j, \frac{3}{2}+v+\frac{1}{2} j
\end{array} ;-x^{2}\right]\right\}
\end{aligned}
$$

for $j=0,1,2, \ldots$.

Again, in (2.1), if we take $p=0, q=1, b_{1}=1+v-j$ and $y=-1$, then

$$
e^{-x} \sum_{n=0}^{\infty} \frac{x^{n} L_{n}^{(v)}(x)}{(1+v-j)_{n}}=\sum_{n=0}^{\infty} \frac{(-x)^{n}}{n !}{ }_{2} F_{1}\left[\begin{array}{c}
-n,-n-v \\
1+v-j
\end{array} ;-1\right] .
$$

The ${ }_{2} F_{1}$ series on the right-hand side of (2.5) can be evaluated with the help of the known result [3]

$$
\begin{aligned}
{ }_{2} F_{1}\left[\begin{array}{c}
a, b \\
1+a-b-j
\end{array} ;-1\right]= & \frac{2^{-a} \Gamma\left(\frac{1}{2}\right) \Gamma(1+a-b-j)}{\Gamma\left(\frac{1}{2} a-b-\frac{1}{2} j+\frac{1}{2}\right) \Gamma\left(\frac{1}{2} a-b-\frac{1}{2} j+1\right)} \\
& \times \sum_{r=0}^{j}\left(\begin{array}{c}
j \\
r
\end{array}\right) \frac{\Gamma\left(\frac{1}{2} a-b-\frac{1}{2} j+\frac{1}{2} r+\frac{1}{2}\right)}{\Gamma\left(\frac{1}{2} a-\frac{1}{2} j+\frac{1}{2} r+\frac{1}{2}\right)}
\end{aligned}
$$

for $j=0,1,2, \ldots$ and, after some simplification, we obtain

$$
\begin{aligned}
S(v,-j) \equiv e^{-x} \sum_{n=0}^{\infty} \frac{x^{n} L_{n}^{(v)}(x)}{(1+v-j)_{n}} \\
=\frac{2^{2 v-j} \Gamma(1+v-j)}{\Gamma(1+2 v-j)} \sum_{r=0}^{j}\left(\begin{array}{c}
j \\
r
\end{array}\right)\left\{\frac{\Gamma\left(v-\frac{1}{2} j+\frac{1}{2} r+\frac{1}{2}\right)}{\Gamma\left(\frac{1}{2}-\frac{1}{2} j+\frac{1}{2} r\right)}\right. \\
\quad \times{ }_{2} F_{3}\left[\begin{array}{c}
\left.\frac{1}{2}+v-\frac{1}{2} j+\frac{1}{2} r, \frac{1}{2}+\frac{1}{2} j-\frac{1}{2} r ;-x^{2}\right] \\
\frac{1}{2}, \frac{1}{2}+v-\frac{1}{2} j, 1+v-\frac{1}{2} j
\end{array}\right] \\
\left.\quad-\frac{4 x \Gamma\left(v-\frac{1}{2} j+\frac{1}{2} r+1\right)}{(1+2 v-j) \Gamma\left(\frac{1}{2} r-\frac{1}{2} j\right)} 2 F_{3}\left[\begin{array}{c}
1+v-\frac{1}{2} j+\frac{1}{2} r, 1+\frac{1}{2} j-\frac{1}{2} r ;-x^{2} \\
\frac{3}{2}, 1+v-\frac{1}{2} j, \frac{3}{2}+v-\frac{1}{2} j
\end{array}\right]\right\}
\end{aligned}
$$

for $j=0,1,2, \ldots$. 


\section{THE SERIES $S(-v, \pm j)$}

Further, if we take $p=0, q=1, b_{1}=1-v+j$ and $y=-1$ in (2.1), we find

$$
e^{-x} \sum_{n=0}^{\infty} \frac{x^{n} L_{n}^{(v)}(x)}{(1-v+j)_{n}}=\sum_{n=0}^{\infty} \frac{(-x)^{n}}{n !}{ }_{2} F_{1}\left[\begin{array}{c}
-n,-n-v \\
1-v+j
\end{array} ;-1\right] .
$$

The ${ }_{2} F_{1}$ series on the right-hand side of (3.1) can be evaluated by (2.3) to produce the result after some simplification

$$
\begin{aligned}
S(-v, j) \equiv e^{-x} \sum_{n=0}^{\infty} \frac{x^{n} L_{n}^{(v)}(x)}{(1-v+j)_{n}} \\
=\frac{(-2)^{j}}{j !} \sum_{r=0}^{j}(-1)^{r}\left(\begin{array}{c}
j \\
r
\end{array}\right)\left\{\frac{\Gamma\left(-\frac{1}{2} v+\frac{1}{2} j+\frac{1}{2} r+\frac{1}{2}\right)}{\Gamma\left(-\frac{1}{2} v-\frac{1}{2} j+\frac{1}{2} r+\frac{1}{2}\right)}\right. \\
\\
\times{ }_{3} F_{4}\left[\begin{array}{c}
1, \frac{1}{2}+\frac{1}{2} v+\frac{1}{2} j-\frac{1}{2} r, \frac{1}{2}-\frac{1}{2} v+\frac{1}{2} j+\frac{1}{2} r \\
\frac{1}{2}+\frac{1}{2} j, 1+\frac{1}{2} j, \frac{1}{2}-\frac{1}{2} v+\frac{1}{2} j, 1-\frac{1}{2} v+\frac{1}{2} j
\end{array} ;-x^{2}\right] \\
-\frac{4 x}{(j+1)(1-v+j)} \frac{\Gamma\left(-\frac{1}{2} v+\frac{1}{2} j+\frac{1}{2} r+1\right)}{\Gamma\left(-\frac{1}{2} v-\frac{1}{2} j+\frac{1}{2} r\right)} \\
\left.\quad \times F_{3}\left[\begin{array}{c}
1,1+\frac{1}{2} v+\frac{1}{2} j-\frac{1}{2} r, 1-\frac{1}{2} v+\frac{1}{2} j+\frac{1}{2} r \\
1+\frac{1}{2} j, \frac{3}{2}+\frac{1}{2} j, 1-\frac{1}{2} v+\frac{1}{2} j, \frac{3}{2}-\frac{1}{2} v+\frac{1}{2} j
\end{array} ;-x^{2}\right]\right\}
\end{aligned}
$$

for $j=0,1,2, \ldots$.

Finally, if we take $p=0, q=1, b_{1}=1-v-j$ and $y=-1$ in (2.1), we find

$$
e^{-x} \sum_{n=0}^{\infty} \frac{x^{n} L_{n}^{(v)}(x)}{(1-v-j)_{n}}=\sum_{n=0}^{\infty} \frac{(-x)^{n}}{n !}{ }_{2} F_{1}\left[\begin{array}{c}
-n,-n-v \\
1-v-j
\end{array} ;-1\right] .
$$

The ${ }_{2} F_{1}$ series on the right-hand side of (3.3) can be evaluated by (2.6) to produce the result after some simplification

$$
\begin{aligned}
& S(-v,-j) \equiv e^{-x} \sum_{n=0}^{\infty} \frac{x^{n} L_{n}^{(v)}(x)}{(1-v-j)_{n}} \\
& =2^{-j} \sum_{r=0}^{j}\left(\begin{array}{l}
j \\
r
\end{array}\right)\left\{{ }_{2} F_{3}\left[\begin{array}{c}
\frac{1}{2}-\frac{1}{2} v-\frac{1}{2} j+\frac{1}{2} r, \frac{1}{2}+\frac{1}{2} v+\frac{1}{2} j-\frac{1}{2} r ;-x^{2} \\
\frac{1}{2}, \frac{1}{2}-\frac{1}{2} v-\frac{1}{2} j, 1-\frac{1}{2} v-\frac{1}{2} j
\end{array}\right]\right. \\
& \left.\quad-\frac{2 x(v+j-r)}{v+j-1}{ }_{2} F_{3}\left[\begin{array}{c}
1-\frac{1}{2} v-\frac{1}{2} j+\frac{1}{2} r, 1+\frac{1}{2} v+\frac{1}{2} j-\frac{1}{2} r ;-x^{2} \\
\frac{3}{2}, 1-\frac{1}{2} v-\frac{1}{2} j, \frac{3}{2}-\frac{1}{2} v-\frac{1}{2} j
\end{array}\right]\right\}
\end{aligned}
$$

for $j=0,1,2, \ldots$. 


\section{CONCLUding REMARKS}

To conclude we make a brief comparison of the results (2.4), (2.7), (3.2) and (3.4) with those obtained in [1]. The summations $S(v, \pm j)$ derived by Brychkov were expressed respectively in terms of finite sums of ${ }_{2} F_{3}\left(-x^{2}\right)$ functions and Bessel functions of the first kind. The summations $S(-v, \pm j)$ were expressed respectively in terms of finite sums of four ${ }_{4} F_{3}\left(-x^{2}\right)$ functions and four ${ }_{6} F_{7}\left(-x^{2}\right)$ functions, including the Jacobi polynomials of zero argument. Our expressions in (3.2) and (3.4) involve simpler finite sums of two ${ }_{3} F_{4}\left(-x^{2}\right)$ and two ${ }_{2} F_{3}\left(-x^{2}\right)$ functions, respectively.

Finally, we mention that the summations $S( \pm v, \pm j)$ have been verified numerically with the help of Mathematica.

\section{ACKNOWLEDGEMENT}

One of the authors (YSK) acknowledges the support of the Wonkwang University Research Fund (2014).

\section{REFERENCES}

[1] Y. A. Brychkov, "Two series containing the Laguerre polynomials," Integral Transforms and Special Functions, vol. 24, no. 11, pp. 911-915, 2013.

[2] Y. S. Kim, A. K. Rathie, and R. B. Paris, "On a new class of summation formulae involving the Laguerre polynomial," Integral Transforms and Special Functions, vol. 23, no. 6, pp. 435-444, 2012.

[3] M. A. Rakha and A. K. Rathie, "Generalizations of classical summation theorems for the series ${ }_{2} F_{1}$ and ${ }_{3} F_{2}$ with applications," Integral Transforms and Special Functions, vol. 22, no. 11, pp. 823-840, 2011.

Authors' addresses

\section{Y. S. Kim}

Department of Mathematics Education, Wonkwang University, Iksan, Korea

E-mail address: yspkim@wonkwang.ac.kr

\section{A. K. Rathie}

Department of Mathematics, Central University of Kerala, Kasaragad 671123, Kerala, India

E-mail address: akrathiedcukerala.edu.in

\section{R. B. Paris}

School of Engineering, Computing and Applied Mathematics, University of Abertay, Dundee DD1 1HG, UK

E-mail address: r.paris@abertay.ac.uk 